\title{
Improvement in the Selection of Patients for Anti-PD1 Immuno- therapy in Non-Small Cell Lung Cancer (NSCLC) Analyzing Tumor Mutational Burden and Retrotransposon Activity as Possible New Biomarker of Effectiveness
}

\author{
Manuel Cobo-Dols* \\ Department of Medical Oncology, Hospital Universitario Málaga Regional y Virgen de la Victoria, Spain
}

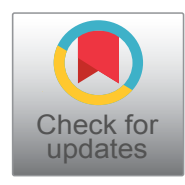

*Corresponding author: Manuel Cobo-Dols, MD, Department of Medical Oncology, Hospital Universitario Málaga Regional y Virgen de la Victoria, IBIMA, 29010 Malaga, Spain, Tel: 661210478, Fax: (+34)952392044, E-mail: manuelcobodols@yahoo.es

Cancer is one of the leading causes of death. Most patients are diagnosed during the late stages of the disease in several tumors. Unfortunately, current treatment options are limited. Habitually, patients receive first- or second-line chemotherapy and a few patients (5-7\%) are candidates for treatment with target agents, that eventually go on to develop resistance to these agents [1]. This explains why the continued search for new therapeutic options is imperative in advanced cancer.

The interaction between the host immune system and cancer has become more evident in recent decades. Mutations have been identified in tumor cells that encode for antigenic peptides present in certain tumor variants. This indicates that immune vigilance of genome integrity in mammals is a reality [2]. Lymphocyte infiltration has been observed in human tumors, and these cells can be reactivated in vitro to destroy cultured tumor cells [3]. This is because patients with cancer can produce T lymphocytes that recognize specific tumor antigens. An important fraction of tumor-specific antigen peptides are produced by genetic anomalies, such as intronic transcription, non-sense transcription, or post-translation [4]. The generation of some tumor-specific antigens depends on the type of transcriptome and proteasome present in the tumor cell [5]. An efficient antitumor activity by the immune system requires adequate functionality and coordination of its effector cells, as are: Cytotoxic CD8 T lym- phocytes (CTL), T helper-1 lymphocytes (Th1), mature dendritic cells (DCS) which activate the inflammatory macrophages (IM), and natural killer cells (NK) [6].

The immune response, be it against either tumor cells or infected cells, can damage healthy tissue if it is not controlled. To protect against this, the immune system has multiple mechanisms to down-regulate the immune response, known collectively as immunological control pathways. Under an immune suppressor situation, tumors use at least three mechanisms to modulate the immune system and avoid detection by immune effector cells: Secretion of soluble immune suppressor factors (e.g., IL-10, TGF- $\beta$, VEGF, etc); interference with the Class I MHC and peptide presentation (via down-regulation of the expression of class I MHC, or the disablement of antigen degradation, or insertion of antigens in the class ( MHC); and contact-dependent factors (expression of checkpoint ligands of the immune system, such as the programmed cell death protein 1 PD-1). All this gives rise to the appearance of T helper cells with the Th2 phenotype, macrophages with the M2 phenotype and regulators LT [6]. Additionally, chronic antigen expression, as occurs in chronic viral infection and cancer, can lead to persistently high expression levels of PD1, which induces a state of exhaustion or anergy in the antigen-specific T cells. Increased expression of PD1 in intratumor CD8+ cells is correlated with this state of anergy or exhaustion $[7,8]$.

Citation: Cobo-Dols M (2018) Improvement in the Selection of Patients for Anti-PD1 Immunotherapy in Non-Small Cell Lung Cancer (NSCLC) Analyzing Tumor Mutational Burden and Retrotransposon Activity as Possible New Biomarker of Effectiveness. Int J Pathol Clin Res 4:068. doi.org/10.23937/24695807/1510068

Accepted: May 28, 2018: Published: May 30, 2018

Copyright: (C) 2018 Cobo-Dols M. This is an open-access article distributed under the terms of the Creative Commons Attribution License, which permits unrestricted use, distribution, and reproduction in any medium, provided the original author and source are credited. 
The presence of specific antigens for different types of tumors explains the three focuses of immunotherapy in cancer, currently under development: Adoptive transfer of antitumor T cells, vaccination, and the use of T-cell stimulating antibodies. The basis of this latter option type of therapy consists of the capacity of T cells to eliminate tumor cells by recognition of aberrant proteins belonging to the cancer cells, and the coordination of an immune response against them [9].

Several immunotherapies have yielded impressive results in the clinic. Recently, the immune modulatory antibodies ipilimumab and pembrolizumab received FDA approval for treatment of metastatic melanoma $[10,11]$, and nivolumab received FDA approval for melanoma and non-small cell lung carcinoma (NSCLC) $[12,13]$. The development of pembrolizumab from the first-in-humans study to FDA approval for the treatment of patients with metastatic melanoma with disease progression following ipilimumab or BRAF inhibitor in BRAF mutation, has occurred in a record 3.6 years. Approval is likely to be extended to include other melanoma populations and other tumor types like NSCLC, head and neck, urotelial, stomach, hodgkin lymphoma and triple-negative breast cancer [14].

No efficient biomarkers have yet been found to individualize this type of therapy in patients with NSCLC or any other type of cancer. Most biomarker investigations for PD-1/PD-L1 agents have focused on the tumor microenvironment and, specifically, immune-histochemical expression of one of the ligands for PD-1, PD-L1. Across all studies in multiple tumor types, it seems that patients whose tumors express PD-L1, as detected by immune-histochemical assays, have numerically higher response rates to PD-1/PD-L1 blockade than patients who do not express PD-L1. In KEYNOTE-001 trial of pembrolizumab in NSCLC, a cutoff of $\geq 50 \%$ PD-L1 positivity in tumor cells was determined as optimal and patients with a score of $\geq 50 \%$ staining $(n=73)$ had a response of $45.2 \%$ (95\% Cl: 33.5-57.3); vs. patients with a score of 1-49\% staining (ORR 15.6\% [95\% Cl: 8.3-25.6]) and $\leq$ $1 \%$ staining (ORR 9.1\% [95\% Cl: 1.1-29.2]). Median PFS in the $\geq 50 \%$ PD-L1-positive group was 6.3 months (95\% Cl: 2.9-12.5), and median OS was not reached [15]. Nevertheless, patients who do not express PD-L1 can still have responses to PD-1 blockade and should be considered eligible for PD-1-blocking approaches. It remains unclear whether differential expression levels of PD-L1 among various tumor types account for the somewhat different response rates observed. Yet, the explanation for the varying response rates to PD-1 blockade among different tumor types is likely more complex. PD-L1 is inducible and can be upregulated in response to infiltrating immune cells and, possibly, genetic changes within the tumor [16].

In addition, the determinants of response to PD-1 blockade appear to be shaped by the genomic alter- ations in tumor cells. With the technological advances in next generation sequencing (NGS), including whole genome sequencing, whole transcriptome sequencing, whole exome sequencing, and targeted sequencing and bioinformatics, our understanding of cancer has dramatically expanded. The genetic and immunologic lines of inquiry are now converging to provide definitive evidence that human cancers are vastly different from normal tissues at the genetic level, and that some of these differences are recognized by the immune system [17].

Tumors develop tens to thousands of coding mutations during the process of tumorigenesis and tumor progression. A small proportion of mutations affect the extracellular domains of cell surface. However, to be recognized by $T$ cells, mutations need to be processed and presented on $\mathrm{MHCl}$ or $\mathrm{MHCll}$, giving rise to so-called "neoantigens" [3]. Most human melanomas have a mutational load above 10 somatic mutations per megabase $(\mathrm{Mb})$ of coding DNA, and this is probably sufficient to lead to the formation of neoantigens that can be seen by $T$ cells. This data is expected to be common for other tumors with a mutational load above 10 somatic mutations per $\mathrm{Mb}$ (corresponding to approximately 150 non-synonymous mutations within expressed genes), like NSCLC, stomach, esophagus, SCLC, urothelial, colorectal with microsatellite instability and uterine carcinoma. Many tumors with a mutational load of 1 to 10 per Mb may still be expected to carry neoantigens [18].

Neoantigens can arise when mutations affect either TCR contact residues or anchor residues in peptide epitopes with affinity for $\mathrm{MHC}$ I or II. Even a single amino acid substitution can yield an epitope that is sufficiently different from self to mark tumor cells for T cell mediated destruction [19]. Whole-exome sequencing of NSCLCs treated with pembrolizumab has shown that higher nonsynonymous mutation burdens in tumors were associated with improved objective responses, durable clinical benefit, and improved PFS. Efficacy also correlates with smoking-induced molecular signatures, higher neoantigen burden due to mutations and DNA repair pathway mutations [20]. Significantly, in colorectal cancer (CRC), observations that somatic mutations due to mismatch-repair (MMR) defects have the potential to encode "non-self" immunogenic antigens suggest that they may be more susceptible to immune checkpoint blockade with pembrolizumab. The MMR-proficient patients was associated with an absence of TIL and relatively low levels of PD-L1 expression, but abundant infiltrating cells in the MMR-deficient patients. Thus, MMR deficiency could be a predictive marker of responses to PD-1 blockade. However, only 4-5\% of all tumors seem to manifest MMR deficiency [21].

The very vastness of genetic changes in cancers now raises different question. Which of the many cancer-specific genetic (genomic) changes are actually recognized by the immune system, and why? New obser- 
vations are now beginning to probe these vital issues with unprecedented resolution and are informing a new generation of studies in human cancer immunotherapy [22]. One of the challenges of research is the roll of active non-coding mobile genetic sequence in immune response against cancer.

LINE-1 (long interspersed nuclear elements) elements are the largest family of human retrotransposons, mobile genetic elements that move in the human genome via an RNA intermediate. The LINE-1 family comprises about 500,000 copies, collectively accounting for as much as $17 \%$ of the human genome. Each LINE-1 copy encodes a bicistronic RNA transcript which is translated into a $40 \mathrm{kDa}$ RNA-binding protein (ORF-1) and a $150 \mathrm{kDa}$ protein (ORF-2), the latter endowed with endonuclease and reverse transcriptase (RT) activities. As such, RT is the most highly repeated protein-coding sequence in the genome of higher eukaryotes and an essential component of the retrotransposition machinery, required not only for the mobilization of its own coding elements, but also for other non-autonomous retrotransposons, such as Alu and SVA [23]. Only in the last decade have roles of the LINE-1-encoded RT been recognized, both in embryogenesis and in tumorigenesis. The RT has emerged as a key regulator of both these processes, in parallel with the increasingly recognized contribution of transposable elements to genome-wide regulatory networks.

The notion that expression of retroelements increases in tumors, while being low in normal tissues, is consistent with recent findings that proteins encoded by the LINE-1 bicistronic open reading frames, i.e. ORF1p and ORF2 $p$, are abundant in a variety of cancers. The activation of the retrotransposition machinery can yield extensive genomic insertions, typical of human cancers. These data confirm that tumors offer a highly permissive environment for retrotransposition, and probably "driver" mutations with a causative potential, favoring the emergence of a typically altered "cancer genome" [24].

Probably, the fact that tumors with high activation of the machinery for retrotransposition, are associated with a potential high and progressive mutational status, the study of the transcript metro assess transpositional activity can provide additional data immunogenicity of tumors and therefore predictive of response to therapy with anti-PD1. In addition, the tumor retrotransposonic activity is probably related to genetic resistance mechanisms of immunotherapy, including the phenomenon of immunoediting, repressing the expression of novel epitopes created or down-regulation of the expression of class I MHC. Therefore, increased of retrotransposonic activity in parallel with the tumor progression to monoclonal Ab anti-PD1 therapy, could justify the association of immunotherapy plus therapies with inhibitory retrotransposonic activity. RT may be pharmacologically inhibited in cancer cell lines using nonnucleoside inhibitors [23] and RNA interference (RNAi) methodology to down-regulate the expression of full-length RT-encoding LINE-1 elements [25]. RT inhibition affects the cancer transcriptome globally and may reduce proliferation of cancer cells and restores their "normal" phenotypes.

Liquid biopsy (analysis of tumor DNA in peripheral blood and (TC), has substantial advantages over solid biopsy, not only for the low amount of biopsy material, but also the feasibility and can be performed at any time during tumor progression with a simple blood test. With liquid biopsy, we have the opportunity to study cancer at every moment of its natural history [26]. With Cell Search system followed by search of fluorescence activated cell sorting (FACS) cells, it has been sequenced in DNA of a single CTC and successfully amplifies its genome [27]. This approach can be reliable when genetic variations are consistently present in several collections of CTC from the same sample, allowing the identification of trunk-mutations. Advances in sequencing systematic studies of CTC have tremendous potential to improve our understanding of the heterogeneity of the evolution of the disease [28].

Probably, it would be useful investigate patients diagnosed with advanced cancer types with high probability of having a high mutational burden, including NSCLC, stomach, esophagus, SCLC, urothelial, colorectal with microsatellite instability and uterine carcinoma to be treated with anti-PD1/PD-L1 therapy. Before treatment, it will be necessary to performer a fresh biopsy and peripheral blood determination for CTC, analyzing exome and transcriptome in both samples and establish a more sensitive predictive model of response to anti-PD1/ PDL1 therapy, respect to PD-L1 analysis by immunohistochemistry. This model will be based on the association of the mutational burden, measured by exome and retrotransposition activity analyzed through transcriptome. In addition, correlate these results between solid and liquid biopsy, analyzing exome and transcriptome in CTC would be useful. Furthermore, at the time of progression to anti-PD1/PD-L1 therapy, a new solid and liquid biopsy will be performed to correlate resistance immunotherapy, with probably increasing retrotransposonic status and if positive correlation, would be justified studies with the combination of both types of therapies.

\section{References}

1. http://www.nccn.org/professionals/

2. Rosenberg SA, Packard BS, Aebersold PM, Solomon D, Topalian SL, et al. (1988) Use of tumor-infiltrating lymphocytes and interleukin 2 in the immunotherapy of patients with metastatic melanoma. A preliminary report. $\mathrm{N}$ Engl J Med 319: 1676-1680.

3. Coulie PG, Van den Eynde BJ, Van der Bruggen P, Boon $T$ (2014) Tumour antigens recognized by $T$ lymphocytes: at the core of cancer immunotherapy. Nature Reviews 14 : 135-146. 
4. Probst-Kepper M, Stroobant V, Kridel R, Gaugler B, Landry C, et al. (2001) An alternative open reading frame of the human macrophage colony-stimulating factor gene is independently translated and codes for an antigenic peptide of 14 amino acids recognized by tumor-infiltrating CD8 T lymphocytes. J Exp Med 193: 1189-1198.

5. Vigneron N, Stroobant V, Chapiro J, Ooms A, Degiovann G, et al. (2004) An antigenic peptide produced by peptide splicing in the proteasome. Science 304: 587-590.

6. Alberto Mantovani, Paola Allavena, Antonio Sica, Frances Balkwill (2008) Cancer-related inflammation. Nature 454 436-444.

7. Pardoll DM (2012) The blockade of immune checkpoints in cancer immunotherapy. Nat Rev Cancer 12: 252-264.

8. Amarnath S, Mangus CW, Wang JCM, Fang Wei, Alice He, et al. (2011) The PDL1-PD1 axis converts human Th1 cells into regulatory T cells. Sci Transl Med 3: 111-120.

9. Whiteside TL (2010) Immune responses to malignancies. J Allergy Clin Immunol 125: S272-S283.

10. (2014) U.S. Food and Drug Administration. FDA.gov.

11. Hodi FS, O'Day SJ, McDermott DF, Weber RW, Sosman JA, et al. (2010) Improved survival with ipilimumab in patients with metastatic melanoma. N Engl J Med 363: 711723.

12. Robert C, Long GV, Brady B, Caroline D, Michele M, et al. (2014) Nivolumab in previously untreated melanoma without BRAF mutation. N Engl J Med.

13. (2015) US Food and Drug Administration. FDA.gov.

14. Khoja L, Butler MO, Kang SP, Ebbinghaus S, Joshua AM (2015) Pembrolizumab. J Immunother Cancer 3: 36.

15. Garon EB, Rizvi NA, Hui R, Leighl N, Balmanoukian AS, et al. (2015) Pembrolizumab for the treatment of non-smallcell lung cancer. N Engl J Med 372: 2018-2028.

16. Taube JM, Klein A, Brahmer JR, Xu H, Pan X, et al. (2014) Association of PD-1, PD-1 ligands, and other features of the tumor immune microenvironment with response to anti-PD-1 therapy. Clin Cancer Res 20: 5064-5074.
17. Abel HJ, Duncavage EJ (2014) Detection of structural DNA variation from next generation sequencing data: a review of informatics approaches. Cancer Genetics 206: 432-440.

18. Schumacher TN, Schreiber RD (2015) Neoantigens in cancer immunotherapy. Science 348: 69-74.

19. Pandha H, Pawelec $G$ (2015) Immune checkpoint targeting as anti-cancer immunotherapy: promises, questions, challenges and the need for predictive biomarkers at ASCO 2015. Cancer Immunol Immunother 64: 1071-1074.

20. Rizvi NA, Hellmann MD, Snyder A, Kvistborg P, Makarov V, et al. (2015) Cancer immunology. Mutational landscape determines sensitivity to PD1 blockade in non-small cell lung cancer. Science 348: 124-128.

21. Le DT, Uram JN, Wang $H$, Bartlett BR, Kemberling $H$, et al. (2015) PD1 blockade in tumors with mismatch-repair deficiency. N Engl J Med 372: 2509-2520.

22. Srivastava PK (2015) Neoepitopes of Cancers: Looking Back, Looking Ahead. Cancer Immunol Res 9: 969-977.

23. Sciamanna I, Gualtieri A, Piazza PF, Spadafora C (2014) Regulatory roles of LINE-1-encoded reverse transcriptase in cancer onset and progression. Oncotarget 5: 8039-8051.

24. Rodić N, Steranka JP, Makohon-Moore A, Allison Moyer, Peilin Shen, et al. (2015) Retrotransposon insertions in the clonal evolution of pancreatic ductal adenocarcinoma. Nature Med 21: 1060-1064.

25. Soifer HS, Zaragoza A, Peyvan M, Behlke MA, Rossi JJ (2005) A potential role for RNA interference in controlling the activity of the human LINE-1 retrotransposon. Nucleic Acids Res 33: 846-856.

26. Alix-Panabieres C, Pantel K (2013) Circulating tumor cells: liquid biopsy of cancer. Clin Chem 59: 110-118.

27. Swennenhuis JF, Reumers J, Thys K, Aerssens J, Terstappen LW (2013) Efficiency of whole genome amplification of single circulating tumor cells enriched by Cell Search and sorted by FACS. Genome Med 5: 106.

28. Wang Y, Waters J, Leung ML, Unruh A, Roh W, et al. (2014) Clonal evolution in breast cancer revealed by single nucleus genome sequencing. Nature 512: 155-160. 